\title{
FORMAÇÃO DO FISIOTERAPEUTA PARA A ATENÇÃO BÁSICA
}

PHYSIOTHERAPIST FORMATION FOR PRIMARY CARE

\section{Liliam Rosany Medeiros Fonseca Barcellos ${ }^{1}$}

1 Fisioterapeuta. Mestre em Atenção à Saúde. Universidade Federal do Triângulo Mineiro. E-mail: lilianmfonseca@ hotmail.com

\section{Natália Lujan Ferraz ${ }^{2}$}

2 Fisioterapeuta. Mestranda em Educação Física. Universidade Federal do Triângulo Mineiro. E-mail: natalialfz@ hotmail.com

\section{Érica Midori Ikegami ${ }^{3}$}

3 Fisioterapeuta. Doutoranda em Atenção à Saúde. Universidade Federal do Triângulo Mineiro. E-mail: erica.ikegami@gmail.com

\section{Lislei Jorge Patrizzi ${ }^{4}$}

4 Fisioterapeuta. Doutora em Ciências Médicas. Universidade Federal do Triângulo Mineiro. E-mail: lispatrizzi@ gmail.com

\section{Isabel Aparecida Porcatti de Walsh $^{5}$}

5 Fisioterapeuta. Doutora em Fisioterapia. Universidade Federal do Triângulo Mineiro.E-mail: ewalsh@terra.com.br

\section{Suraya Novais Gomes Shimano ${ }^{6}$}

6 Fisioterapeuta. Doutora em Ciências da Reabilitação. Universidade Federal do Triângulo Mineiro. E-mail: surayagnovais@gmail.com

\section{RESUMO}

O presente estudo teve como objetivo analisar o preparo para atuação na Atenção Básica $(A B)$, por parte de graduandos do último ano e docentes do curso de Fisioterapia de Instituições de Ensino Superior (IES) pública e privadas de uma cidade de médio porte de Minas Gerais. Pesquisa transversal realizada por meio de entrevistas individuais com 64 graduandos e 28 docentes que responderam um questionário semiestruturado com aspectos sobre a atuação do fisioterapeuta para a $A B$. Os resultados revelaram que os graduandos acreditam que as disciplinas cursadas relacionadas a AB foram suficientes para atuar nesse nível de atenção, ao contrário dos docentes, que acreditam que seus discentes não estão aptos para intervir. A opinião dos graduandos que julgam o tempo de estágio na $A B$ adequado foi bem próxima daqueles que não. Referente as atividades extraclasse relacionadas a $A B$, essas ainda são escassas. Os docentes conseguem fazer associações entre as disciplinas que ministra com a $A B$ e durante sua formação tiveram disciplinas relacionadas, mas essas não forneceram bases para a prática. Espera-se que os achados contribuam para o direcionamento de ações no campo acadêmico, visando a proposição de mudanças nos currículos dos cursos, ampliação do conhecimento sobre esse nível de atenção e auxílio na formação de um profissional em consonância com os princípios do SUS e da $A B$, rompendo com o caráter estritamente reabilitador da profissão.

PALAVRAS-CHAVE: Fisioterapeutas. Capacitação de recursos humanos em saúde. Atenção primária à saúde. 


\section{ABSTRACT}

The present study had as objective to analyze the preparation for action in Primary Care (PC) by undergraduate students of the last year and professors of the physiotherapy course of Institutions of Higher Education (IHE) of public and private sectors of a medium-sized city of Minas Gerais. Cross - sectional research was carried out through individual interviews with 64 undergraduates and 28 teachers who answered a semi structured questionnaire with aspects about the physiotherapist's performance for PC. The results showed that undergraduates believe that the disciplines studied related to PC were sufficient to act at this level of attention, unlike the teachers, who believe that their students they are not able to intervene. The opinion of graduates judging the appropriate internship time in PC was very close to those who did not. Regarding extraclass activities related to $\mathrm{PC}$, these are still scarce. Teachers are able to make associations between the disciplines that they minister with PC and during their formation had related disciplines, but these did not provide bases for the practice. It is hoped that the findings contribute to the direction of actions in the academic field, aiming at proposing changes in the curricula of the courses, expanding knowledge about this level of attention and assisting in the formation of a professional in harmony with the principles of the Single System of Health (SSH) and PC, breaking with the strictly rehabilitating character of the profession.

KEYWORDS: Physical therapists. Health human resource raining. Primary health care.

\section{INTRODUÇÃO}

A promulgação da Constituição Federal de 1988 apresentou as bases para a criação do Sistema Único de Saúde (SUS) no Brasil, pautado nos princípios da universalidade, integralidade e participação social. O sistema foi criado em um contexto socioeconômico e político desfavorável e, ainda está enfrentando desafios no que se refere a gestão, acesso aos serviços, cobertura das ações, estrutura, financiamento e mudanças no modelo de atenção (PAIM et al., 2011).

A Constituição Federal definiu como uma das atribuições do SUS, o ordenamento da formação de recursos humanos (BRASIL, 1988), entretanto, apesar de ser uma ação necessária, a formação dos profissionais de saúde ainda é uma questão preocupante no contexto da implantação do sistema público de saúde no Brasil (GONÇALVES; CARVALHO; TRELHA, 2012).

A necessidade de modificar a forma de atuação no processo de construção e implantação do SUS é uma realidade colocada para profissionais de diversas áreas do conhecimento, dentre eles, o fisioterapeuta, que ao se deparar com novas demandas deve romper o caráter predominantemente reabilitador da profissão e abranger práticas de prevenção de doenças e promoção da saúde (ALMEIDA; MARTINS; ESCALDA, 2014).

A Atenção Básica (AB) é a principal porta de entrada para o SUS e abrange um conjunto de ações de promoção da saúde, prevenção de agravos, diagnóstico, tratamento e reabilitação; realizadas pela equipe multiprofissional, visando a atenção integral do indivíduo (BRASIL, 2017). O fisioterapeuta, enquanto integrante dessa equipe possui capacidade para atuar em todos os níveis de atenção e, recentemente, verifica-se a crescente preocupação em uma formação profissional que contemple o perfil 
para a atuação na AB (RIBEIRO; FLORES-SOARES, 2015).

A profissão de Fisioterapia como nível superior foi instituída em 1969 pelo Decreto-Lei no 938/69 (BRASIL, 1969). Quando a profissão surgiu, o fisioterapeuta era considerado um profissional de nível técnico e sua atuação se restringia a execução de técnicas contidas nas prescrições médicas, de caráter meramente reabilitador para um único público, ou seja, aqueles que apresentassem lesões. Após a publicação do decreto, mesmo com a aquisição de autonomia, a Fisioterapia ainda carregava o perfil reabilitador, entretanto, com as transformações epidemiológicas e a implantação do SUS, novos desafios foram apresentados a profissão (BISPO JÚNIOR, 2010).

A necessidade de capacitação orientou a reformulação das bases da formação dos profissionais de saúde no Brasil. Nesse sentido, em 2001, o Ministério da Educação e Cultura (MEC) instituiu as Diretrizes Curriculares Nacionais (DCN) do Curso de Graduação em Fisioterapia. Segundo a resolução, o fisioterapeuta deve ser capaz de atuar em todos os níveis de atenção à saúde, em programas de promoção, manutenção, prevenção, proteção e recuperação da saúde (BRASIL, 2002).

Os processos de formação de profissionais de saúde no contexto das Instituições de Ensino Superior (IES) e na inserção no mercado de trabalho passaram por transformações desde o surgimento das DCN, devido a necessidade de alcançar um perfil profissional que atenda as demandas impostas pela realidade social do país (PIMENTEL; SILVA; LIMA NETO, 2016).

Em 2017, o Conselho Federal de Fisioterapia e Terapia Ocupacional (COFFITO) publicou o esboço de minuta das DCN do curso de graduação, bacharelado, em Fisioterapia; destacando como princípios de formação do fisioterapeuta: o SUS, a saúde como direito fundamental do ser humano, a integralidade da atenção e a promoção da saúde (COFFITO, 2017).

Entretanto, mesmo com o incentivo de ações visando a proposição de mudanças curriculares e o estímulo por parte do governo para implementação de programas, projetos e atividades voltadas para a formação universitária e o trabalho no SUS, verifica-se que o ensino em Fisioterapia ainda não está em consonância com os princípios do sistema de saúde, como a integralidade (ALMEIDA; MARTINS; ESCALDA, 2014) que também é preconizada pela $A B$.

Estudos de revisão da literatura mostram que as pesquisas no Brasil se concentram na análise da inserção, das atividades desenvolvidas e sobre a importância do fisioterapeuta na $A B$, destacando sua contribuição para o trabalho e a difusão desse nível de atenção. Entretanto, mesmo com os avanços, a prática do fisioterapeuta na $A B$ ainda é incipiente e voltada para práticas assistenciais (MAIA et al., 2015; FONSECA et al., 2016; SILVA; ALMEIDA, 2016).

Verifica-se que a investigação da formação profissional do fisioterapeuta para a atuação na $A B$ ainda é pouco explorada e que existe a necessidade de ampliar as práticas desse profissional além do campo da reabilitação. Dessa forma, o objetivo desse estudo foi analisar o preparo para atuação na $A B$, por parte de graduandos do último ano e docentes do curso de Fisioterapia de Instituições de Ensino Superior (IES) pública e privadas de uma cidade de médio porte de Minas Gerais.

\section{MATERIAIS E MÉTODOS}

Trata-se de um estudo transversal realizado em uma cidade de médio porte de Minas Gerais, em três instituições de ensino superior que oferecem o curso de Fisioterapia: A (privada), B (privada) e C (pública). 
Os participantes foram abordados de agosto a setembro de 2016 e a amostra de conveniência foi composta por 64 graduandos do último ano e 28 docentes. Os critérios de inclusão adotados foram: ser aluno ou docente do curso de Fisioterapia e apresentar confirmação da matrícula (graduandos) ou o coordenador afirmar que faz parte do quadro de alunos ou docentes da instituição.

Todos os participantes que aceitaram participar da pesquisa assinaram o Termo de Consentimento Livre e Esclarecido. Procedeu-se a entrevista individual com a aplicação de um questionário semiestruturado (Quadro 1), composto de perguntas elaboradas pelas autoras a partir do levantamento de dados da literatura especializada que abrangiam aspectos sobre a atuação do fisioterapeuta para a AB (ALVES; RIBEIRO; MAIA FILHO, 2016; SOUZA et al., 2014; PIMENTEL; SILVA; LIMA NETO, 2016; SERIANO; MUNIZ; CARVALHO, 2013).

Quadro 1 - Questionário com questões referentes a formação do fisioterapeuta para $a A B$

\begin{tabular}{|l|}
\hline Questões comuns a todos os entrevistados \\
\hline Instituição de Ensino Superior a que pertence \\
\hline Idade \\
\hline Sexo ( ) Masculino ( ) Feminino \\
\hline Questões específicas para graduandos \\
\hline $\begin{array}{l}\text { As disciplinas cursadas relacionadas a AB foram suficientes para a atuação nesse nivel de atenção? } \\
\text { ( ) Sim ( ) Não }\end{array}$ \\
\hline O tempo de estágio na AB é adequado para a atuação nesse nível de atenção? ( ) Sim ( ) Não \\
\hline Realizou alguma atividade extraclasse relacionada com a AB? ( ) Sim ( ) Não \\
\hline Questões específicas para os docentes \\
\hline Tem quantos anos de formação? \\
\hline Quantas disciplinas ministra atualmente? \\
\hline Durante a sua formação havia disciplinas relacionadas a AB? ( ) Sim ( ) Não \\
\hline As disciplinas cursadas foram suficientes para a atuação na AB? ( ) Sim ( ) Não \\
\hline Consegue fazer associações entre as disciplinas que ministra com a AB? ( ) Sim ( ) Não \\
\hline Os graduandos estão aptos para atuarem na AB? ( ) Sim ( ) Não \\
\hline Fonte: Das autoras, 2016.
\end{tabular}

Os dados foram digitados em planilha eletrônica do programa Microsoft Office Exce $^{\oplus} \mathrm{e}$ foi realizada a checagem de consistência dos dados, que em seguida foram analisados no programa Statistical Package for the Social Sciences (SPSS) versão 21.0. Foi realizada análise estatística descritiva, através de frequências absolutas e relativas para as variáveis qualitativas; e medidas de tendência central (média) e de variabilidade (desvio padrão) para as variáveis quantitativas.

A coleta de dados da pesquisa foi iniciada após aprovação pelo Comitê de Ética e Pesquisa com Seres Humanos da Universidade Federal do Triângulo Mineiro (CEP - UFTM), em obediência à resolução 466/12 do Conselho Nacional de Saúde, com aprovação sob o parecer no 1.697.459. 


\section{RESULTADOS}

Entre os participantes, a média de idade foi de 19,42 $\pm 9,42$ anos para os grad-

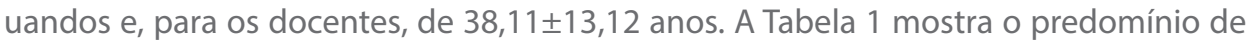
alunos na instituição C (46,9\%) e de docentes nas instituições B e C, com 35,7\% cada. Em relação ao sexo, houve predomínio de mulheres tanto entre os graduandos (95,3\%) quanto docentes $(67,9 \%)$.

Tabela 1 - Distribuição das variáveis IES e sexo entre graduandos e docentes. Uberaba, Minas Gerais, 2016.

\begin{tabular}{lcccc} 
Variável & \multicolumn{2}{c}{ Graduandos } & \multicolumn{2}{c}{ Docentes } \\
\cline { 2 - 5 } & $\mathbf{n}$ & $\%$ & $\mathbf{n}$ & \% \\
\cline { 2 - 5 } Instituição de Ensino Superior & 10 & 15,6 & 8 & 28,6 \\
Instituição A & 24 & 37,5 & 10 & 35,7 \\
Instituição B & 30 & 46,9 & 10 & 35,7 \\
Instituição C & & & & \\
Sexo & 3 & 4,7 & 9 & 32,1 \\
Masculino & 61 & 95,3 & 19 & 67,9 \\
Feminino & &
\end{tabular}

Fonte: Dados resultantes da pesquisa, 2016.

Conforme da Tabela 2, na primeira questão destinada aos graduandos, 62,5\% afirmou que as disciplinas cursadas foram suficientes para atuar na AB. Quando questionados se o tempo de estágio na $A B$ era adequado para atuação nesse nível de atenção, $37,5 \%$ respondeu que não. Referente as atividades extraclasses relacionadas com a $A B$, a maioria relatou não ter realizado durante a graduação $(40,6 \%)$

Tabela 2 - Distribuição de graduandos quanto ao tempo de estágio e atividades extraclasse relacionadas com a AB. Uberaba, Minas Gerais, 2016

\begin{tabular}{lcc} 
Pergunta & $\mathbf{n}$ & $\%$ \\
\cline { 2 - 2 } $\begin{array}{l}\text { As disciplinas cursadas relacionadas a AB foram suficientes } \\
\text { para a atuação nesse nível de atenção? }\end{array}$ & \\
Não respondeu & 5 & 7,8 \\
Sim & 40 & 62,5 \\
Não & 19 & 29,7 \\
O tempo de estágio na AB é adequado para a atuação nesse & & \\
nível de atenção? & 15 & 23,4 \\
Não respondeu & 25 & 39,1 \\
Sim & 24 & 37,5 \\
Não & & 32,8 \\
Realizou alguma atividade extraclasse relacionada com a AB? & 21 & 26,6 \\
Não respondeu & 17 & 40,6 \\
Nim & 26 & \\
\hline
\end{tabular}

Fonte: Dados resultantes da pesquisa, 2016.

Concernente as questões específicas ao docente, a média de anos de formação

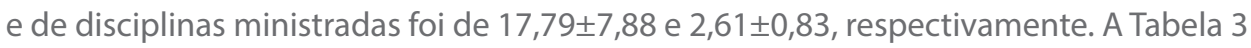
mostra que $60,7 \%$ tiveram disciplinas relacionadas a $A B$ durante sua formação, entretanto, essas não foram suficientes para atuação nesse nível de atenção (50\%). Apesar dos docentes conseguirem realizar associações entre suas disciplinas e a $A B(85,7 \%)$, eles acreditam que os alunos não estão aptos para atuarem na $\mathrm{AB}(50 \%)$. 


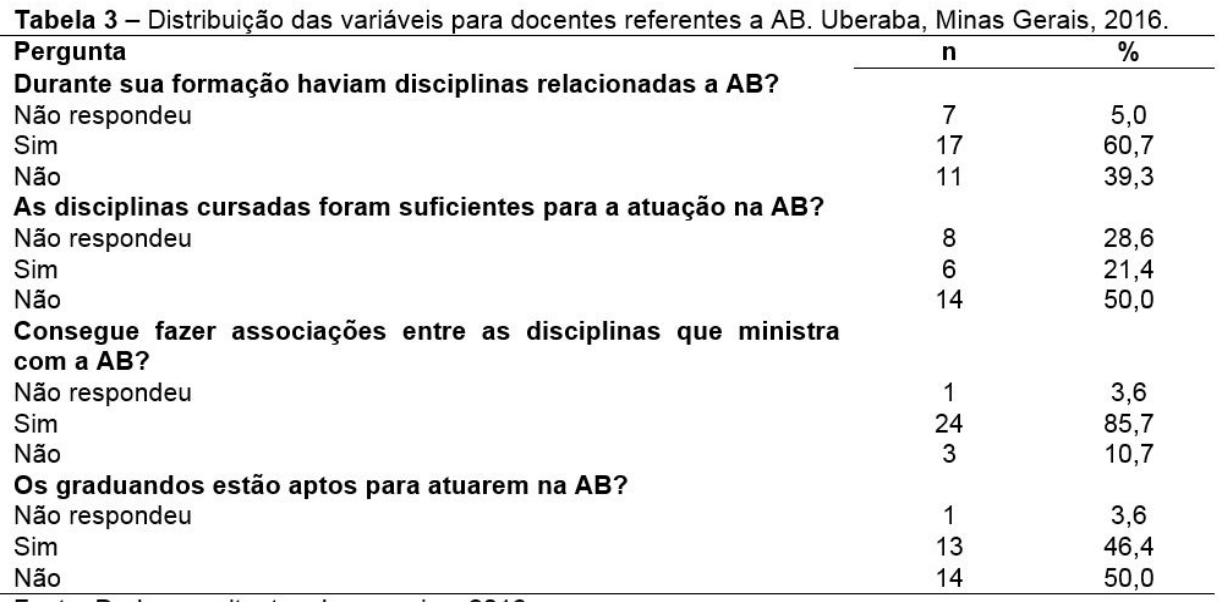

Fonte: Dados resultantes da pesquisa, 2016.

\section{DISCUSSÃO}

Os graduandos entrevistados acreditam que as disciplinas cursadas relacionadas a $A B$ são suficientes para atuar nesse nível de atenção. $O$ achado é corroborado pelos resultados de estudo realizado com graduandos do $6^{\circ}$ ao $10^{\circ}$ período de Fisioterapia da Universidade Estadual do Piauí, onde os mesmos afirmaram que a IES proporcionou conhecimentos sobre o SUS durante a vida acadêmica e que se sentem preparados para atuar no sistema (SERIANO; MUNIZ; CARVALHO, 2013).

Estudo realizado com estudantes do $7^{\circ}, 8^{\circ}$ e $9^{\circ}$ período de uma IES particular mostra resultados divergentes do presente estudo, onde a maioria relatou não se sentir preparado para a atuação. Entretanto, a pesquisa mostrou que o relato de falta de preparo por parte dos estudantes do $8^{\circ}$ e $9^{\circ}$ período é menor quando comparado ao $7^{\circ}$ período, evidenciando a importância das instituições em proporcionar atividades que contemplem as diversas faces da profissão com ampliação do conhecimento teórico e prático (ALVES; RIBEIRO; MAIA FILHO, 2016).

Entretanto, quando foram questionados sobre o tempo de estágio na $A B$ ser adequado para atuar nesse nível de atenção, verificou-se resultados próximos entre os que consideram ou não o período satisfatório. Deve-se ter cautela para a interpretação desse achado, visto que porcentagem expressiva de graduandos não respondeu à questão (23,4\%). Independentemente desse aspecto, verifica-se que o estágio supervisionado é uma vivência indispensável ao graduando.

Estudo realizado em Teresina, em um curso de Fisioterapia de uma IES privada mostrou que $68 \%$ dos graduandos não tiveram vivência na $A B$ nos estágios de saúde coletiva e Fisioterapia comunitária (ALVES; RIBEIRO; MAIA FILHO, 2016), aproximando-se a realidade do presente estudo. $O$ estágio supervisionado proporciona a associação da teoria com a prática, na qual se inclui o exercício do trabalho em equipe, ou seja, os graduandos têm a oportunidade de conhecer os funcionários da instituição e aproximar dos colegas e, principalmente de se descobrir como profissionais através do desenvolvimento de habilidades e relacionamentos (EVANGELISTA; IVO, 2014).

Ainda sobre os estágios supervisionados, outro estudo também realizado em Teresina, porém, em IES pública, revelou que a vivência dos graduandos do $6^{\circ}$ ao $10^{\circ}$ período com o atendimento pelo SUS se concentrou no nível terciário $(78,6 \%)$, enquanto na $A B$ (saúde coletiva e Fisioterapia comunitária) foram apenas 28,6\% (SERIANO; MU- 
NIZ; CARVALHO, 2013). A escassa vivência em estágios supervisionados na AB pode ser justificada pela própria origem da profissão pautada no modelo assistencial curativo, o que restringe a prática profissional do fisioterapeuta ao contexto da doença já instalada e da necessidade de reabilitação (BISPO JÚNIOR, 2010).

Concernente a realização de atividades extraclasse relacionadas a $A B$, constatou-se que 40,6\% não teve a vivência. Entretanto, semelhante a questão sobre o tempo de estágio, as atividades extraclasses devem ser analisadas com cuidado, visto que $32,8 \%$ dos participantes não responderam essa pergunta. Independentemente dos que não responderam, verifica-se que a vivência proporcionada além da sala de aula relacionadas a $\mathrm{AB}$ ainda é escassa.

A diversidade de experiências proporcionadas ao graduando permite o desenvolvimento da compreensão sobre o trabalho a ser realizado em qualquer nível de atenção e a articulação da teoria com a prática, resultando em uma formação generalista pautada na integralidade por meio de vivências extramuros com usuários e famílias (GAUER et al., 2018).

Uma das estratégias para o incentivo de práticas na $A B$ é o Programa de Educação pelo Trabalho para a Saúde (PET-Saúde/GraduaSUS) criado em 2008 em parceria com o Ministério da Saúde e Ministério da Educação, que trouxe a proposta de educação pelo trabalho voltado para graduandos e pós-graduandos da área da saúde com objetivo de promover a qualificação em serviço dos profissionais de acordo com as necessidades do SUS e contribuir para a implementação das Diretrizes Curriculares Nacionais dos cursos de graduação da área da saúde (BRASIL, 2010).

Os anos de formação dos docentes do presente estudo (17,79 $\pm 7,88$ anos) divergem de estudo realizado em João Pessoa, onde foi identificado o predomínio de profissionais com cinco a dez anos de formação (53,85\%). A qualidade do ensino oferecido aos graduandos pode ser influenciada pelo tempo de formação do docente, no que concerne a experiência acumulada no decorrer dos anos, que contribui para a prática educativa no processo ensino-aprendizagem (PIMENTEL; SILVA; LIMA NETO, 2016).

Em relação a formação profissional, os docentes afirmaram que durante esse processo tiveram o contato com disciplinas relacionadas a AB. A implantação das DCN do curso de Fisioterapia e o avanço no conhecimento sobre a importância da inserção desse profissional na $A B$ colocou em evidência a necessidade de desenvolver competências para atuar nesse nível de atenção, que vem sendo proporcionada pela mudança nos currículos dos cursos de graduação com a inclusão de disciplinas e estágios na AB (BATISTON et al., 2017), o que pode justificar o contato relatado pelos docentes.

Apesar do contato com as disciplinas cursadas relacionadas a $A B$, estas não foram consideradas suficientes para a atuação nesse nível de atenção, ao contrário da opinião dos alunos. Sugere-se que isso ocorra em partes, devido a segregação da teoria e da prática característica do treinamento prático tradicional experenciado nas universidades, onde o conhecimento é apresentado de forma irrelevante, hierarquizado e desintegrado (FERREIRA; FIORINI; CRIVELARO, 2010).

Apesar das DCN estabelecidas em 2002 terem impulsionado a inserção de atividades acadêmicas relacionadas a $A B$ e levantado a necessidade de uma formação generalista para promover ações em todos os níveis de atenção, verifica-se que a atuação do fisioterapeuta nesse nível de atenção ainda é relativamente nova (GONÇALVEZ; CARVALHO; TRELHA, 2012). Apesar da incorporação de disciplinas e estágios nos currículos, verifica-se que a inserção do fisioterapeuta na $A B$ ainda é incipiente, o que dificulta a vivência do graduando com a realidade desse profissional (BATISTON et al., 
2017).

Entretanto, mesmo as disciplinas não sendo suficientes para a atuação nesse nível de atenção, os docentes conseguem fazer associações entre as disciplinas que ministra com a AB. Estudo realizado em uma IES da rede privada mostrou que a formação do fisioterapeuta abrange escassas práticas interdisciplinares, o que pode favorecer a fragmentação do saber e prejudicar o desenvolvimento da integralidade do cuidado prestado (CARVALHO; TOMAZ; TAVARES, 2018).

A capacidade do docente em fazer associações entre as disciplinas que ministra com a $A B$ contradiz achado de estudo que analisou as percepções e opiniões de graduandos de Fisioterapia em relação a integralidade da assistência, onde a falta de articulação entre as disciplinas foi encontrada, evidenciando a dificuldade ou negligência dos cursos quanto a reestruturação curricular (ALMEIDA; MARTINS, ESCALDA, 2014).

Quando os docentes foram questionados se seus alunos estão aptos para a atuação na $A B$, a opinião dos que acreditam que sim e dos que julgam que não foram próximas. Estudo que avaliou o processo formativo do curso de Fisioterapia revelou que os docentes acreditam que o nível de atenção à saúde que o fisioterapeuta menos se prepara durante a formação é o primário, sendo a maior ênfase para o terciário (PIMENTEL; SILVA; LIMA NETO, 2016), justificando o achado do presente estudo. Embora a Fisioterapia tenha se aperfeiçoado e ampliado o rol de técnicas e conhecimentos nos últimos anos, verifica-se que essa evolução se concentrou predominantemente em nível terciário, restringindo a prática do profissional aos hospitais e clínicas de reabilitação em pacientes com doença já instalada (BISPO JÚNIOR, 2010).

Considerando que os docentes não acreditam que seus discentes estão aptos para a atuação na $A B$ é necessário destacar o papel do docente na formação profissional. As DCN trazem no seu texto a importância da Educação Permanente dos profissionais para a sua própria formação e prática, mas principalmente para o compromisso com a educação e treinamento/estágio das gerações futuras (BRASIL, 2002). O docente deve inovar a proposta de ações no processo ensino-aprendizagem incluindo saberes e práticas que valorizem a formação de um profissional participativo, questionador e que atenda as necessidades da comunidade (FERREIRA; FIORINI; CRIVELARO, 2010).

Os currículos de Fisioterapia devem adotar a $A B$ como sua base conceitual, visando a recepção e adequação das necessidades dos serviços de saúde e as iniciativas do governo e da sociedade e, principalmente garantir que os futuros profissionais também se adequem as finalidades da $A B$, por meio da inserção de práticas nesse cenário de atuação, foco em ações de promoção da saúde, no trabalho interdisciplinar e no desenvolvimento de habilidades de comunicação e abordagem de métodos de ensino inovadores durante todo o processo de formação (MCMAHON et al., 2016).

\section{CONSIDERAÇÕES FINAIS}

O presente estudo analisou o preparo para atuação na $A B$, por parte de graduandos do último ano e docentes do curso de Fisioterapia. Verificou-se que os graduandos acreditam que as disciplinas cursadas relacionadas a $A B$ foram suficientes para atuar nesse nível de atenção, ao contrário dos docentes, que consideram que seus discentes não estão aptos para intervir.

$O$ estudo mostrou ainda que a opinião dos graduandos que julgam o tempo de estágio na $A B$ adequado foi bem próxima daqueles que não. Referente as atividades extraclasse relacionadas a $A B$, essas ainda são escassas. Os docentes relataram que conseguem fazer associações entre as disciplinas que ministra com a $A B$ e que durante sua 
formação tiveram disciplinas relacionadas, mas que essas não forneceram bases para a prática.

Dessa forma, verifica-se que existe a necessidade de rever a prática e o diálogo entre docentes, graduandos, IES e instâncias governamentais, valorizando o incentivo não somente ao ensino, mas a pesquisa, extensão, atividades extraclasse e os diferentes campos de atuação na AB. A promoção de parcerias entre as IES e secretarias de saúde deve ser priorizada, com objetivo de proporcionar o contato dos graduandos com a $A B$ já no início do processo de formação profissional.

Espera-se que os achados do presente estudo contribuam para o direcionamento de ações no campo acadêmico, não só da Fisioterapia, visando a proposição de mudanças nos currículos dos cursos e a ampliação do conhecimento sobre esse nível de atenção, mas principalmente, auxiliem na formação de um profissional em consonância com os princípios do SUS e da $A B$, rompendo com o caráter estritamente reabilitador da profissão.

\section{REFERÊNCIAS}

ALMEIDA, S. M.; MARTINS, A. M.; ESCALDA, P. M. Integralidade e formação para o Sistema Único de Saúde na perspectiva de graduandos em Fisioterapia. Fisioterapia e Pesquisa, v. 21, n. 2, p. 271-278, 2014.

ALVES, H. N. S.; RIBEIRO, M. T. G.; MAIA FILHO, A. L. M. Percepção de estudantes do curso de fisioterapia de uma instituição privada sobre sua formação profissional para atuação no Sistema Único de Saúde. Revista Saúde em Foco, v. 3, n. 1, p. 20-35, 2016.

BATISTON, A. P. et al. Implantação de uma nova proposta pedagógica para o estágio supervisionado em Fisioterapia na Atenção Básica: relato de experiência. Cadernos de Educação, Saúde e Fisioterapia, v. 4, n. 8, p. 49-55, 2017.

BISPO JÚNIOR, J. P. Fisioterapia e saúde coletiva: desafios e novas responsabilidades profissionais. Ciência \& Saúde Coletiva, v. 15, p. 1627-1636, 2010.

BRASIL. Decreto Lei 938 de 13 de outubro de 1969. Provê sobre as profissões de fisioterapeuta e terapeuta ocupacional e dá outras providências. Diário Oficial da União, Brasília, DF, 14 out. 1969. Disponível em:< http://www.planalto.gov.br/ccivil_03/decreto-lei/1965-1988/Del0938.htm>. Acesso em: 28 jan. 2017.

BRASIL. Constituição da República Federativa do Brasil de 05 de outubro de 1988. Diário Oficial da República Federativa do Brasil. Título VIII. Da Ordem Social. Capítulo II. Seção II. Da Saúde. Arts 196-200. Brasília, DF, 1988. Disponível em: <http://www. planalto.gov.br/ccivil_03/constituicao/constituicaocompilado.htm>. Acesso em: 26 jan. 2017.

BRASIL. Conselho Nacional de Educação Superior. Resolução CNE/CES nº 4, de 19 de fevereiro de 2002. Institui Diretrizes Curriculares Nacionais do Curso de Graduação em Fisioterapia. Disponível em:< http://portal.mec.gov.br/cne/arquivos/pdf/ CES042002.pdf>. Acesso em: 25 jan. 2017. 
BRASIL. Ministério da Saúde. Portaria Interministerial n 421, de 03 de março de 2010. Institui o Programa de Educação pelo Trabalho para a Saúde (PET-Saúde) e dá outras providências. Diário Oficial da União. 2010. Disponível em:< http://bvsms. saude.gov.br/bvs/saudelegis/gm/2010/pri0421_03_03_2010.html>. Acesso em: 30 jan. 2018.

BRASIL. Ministério da Saúde. Portaria n².436, de 21 de setembro de 2017. Aprova a Política Nacional de Atenção Básica, estabelecendo a revisão de diretrizes para a organização da Atenção Básica, no âmbito do Sistema Único de Saúde (SUS). Brasília, DF, 2017. Disponível em: <http://www.brasilsus.com.br/index.php/legislacoes/gabinete-do-ministro/16247-portaria-n-2-436-de-21-de-setembro-de-2017>. Acesso em: 30 abr. 2019.

CARVALHO, V. L.; TOMAZ, J. M. T.; TAVARES, C. H. F Interprofissionalismo e interdisciplinaridade na formação acadêmica: a percepção dos formandos em fisioterapia. Journal of Nursing UFPE online, v. 12, n.4, p. 908-915, 2018.

COFFITO. Conselho Federal de Fisioterapia e Terapia Ocupacional. Esboço de Minuta das Diretrizes Curriculares Nacionais do curso de graduação, bacharelado, em Fisioterapia. Brasília, 2017. Disponível em: <http://coffito.gov.br/nsite/wp-content/ uploads/2017/05/ESBOC\%CC\%A7O-MINUTA-CORRIGIDO-Fisioterapia.pdf>. Acesso em: 13 mar. 2018.

EVANGELISTA, D. L.; IVO, O. P. Contribuições do estágio supervisionado para a formação do profissional de enfermagem: expectativas e desafios. Revista Enfermagem Contemporânea. v. 3, n. 2, p. 123-130, 2014.

FERREIRA, R. C.; FIORINI, V. M. L.; CRIVELARO, E. Formação profissional no SUS: o papel da Atenção Básica em Saúde na perspectiva docente. Revista brasileira de educação médica, v. 34, n. 2, p. 207-2015, 2010.

FONSECA, J. M. A. et al. A Fisioterapia na Atenção Primária à Saúde: uma revisão integrativa. Revista Brasileira em Promoção da Saúde, v. 29, n. 2, p. 288-294, 2016.

GAUER, A. P. M. et al. Ações de reorientação da formação profissional em Fisioterapia: enfoque sobre cenários de prática. Interface - Comunicação, Saúde, Educação, v. 22, n. 65, p.565-576, 29 jun. 2017.

GONÇALVES, F. G.; CARVALHO, B. G.; TRELHA, C. S. O ensino da Saúde Coletiva na Universidade Estadual de Londrina: da análise documental à percepção dos estudantes. Trabalho Educação e Saúde, v. 10 n. 2, p. 301-314, 2012.

MAIA, F. E. S. et al. A importância da inclusão do profissional Fisioterapeuta na atenção básica de saúde. Revista da Faculdade de Ciências Médicas de Sorocaba, v. 17, n. 3, p. 110-115, 2015.

McMAHON, S. et al. Standing on the Precipice: Evaluating Final-Year Physiotherapy Students' Perspectives of Their Curriculum as Preparation for Primary Health Care Practice. Physiother Canada, v. 68 n. 2, p. 188-196, 2016. 
PAIM, J. et al. O sistema de saúde brasileiro: história, avanços e desafios. Lancet. (Série Brasil). p. 11-31, 2011. Disponível em: <http://download.thelancet.com/flatcontentassets/pdfs/brazil/brazilpor1.pdf>. Acesso em: 25 abr. 2019.

PIMENTEL, D. M.; SILVA, C. C.; LIMA NETO, E. A. Bases metodológicas da formação em Fisioterapia: discutindo o distanciamento entre os processos de formação e a utilização da força de trabalho. Tempus Actas de Saúde Coletiva, v. 10, n. 2, p.47-65, 7 jul. 2016.

RIBEIRO, C. D.; FLORES-SOARES, M. C. Desafios para a inserção do fisioterapeuta na atenção básica: o olhar dos gestores. Revista de Salud Pública, v. 17, n. 3, p.379-393, 18 nov. 2015.

SILVA, C. V. P.; ALMEIDA, M. M. C. Fisioterapia e saúde coletiva: uma análise crítica da produção científica no Brasil. Cadernos de Educação, Saúde e Fisioterapia, v. 3, n. 5, p. 32-40, 2016.

SERIANO, K. N.; MUNIZ, V. R. C. M.; CARVALHO, M. E. I. M. Percepção de estudantes do curso de fisioterapia sobre sua formação profissional para atuação na atenção básica no Sistema Único de Saúde. Fisioterapia e Pesquisa, v. 20, n. 3, p. 250-255, 2013.

SOUZA, M. C. et al. Formação acadêmica do fisioterapeuta para atenção básica. Revista UNILUS Ensino e Pesquisa, v. 11, n. 23, p. 59-69, 2014.

Recebido em: 27-03-2018

Aceito em: 01-07-2019 